\title{
Guaranteed cost control for exponential stability of a nonlinear system with mixed time-varying delays in states and controls
}

\author{
Nuchira Khongja ${ }^{1}$, Thongchai Botmart ${ }^{1 *}$, Piyapong Niamsup ${ }^{2}$ and Wajaree Weera ${ }^{3}$
}

\section{"Correspondence:}

thongbo@kku.ac.th

'Department of Mathematics, Khon

Kaen University, Khon Kaen,

Thailand

Full list of author information is

available at the end of the article

\section{Springer}

\begin{abstract}
This paper presents a new guaranteed cost control for exponential stability of a nonlinear system with mixed time-delays in state and feedback control. The considered mixed time-delays are both discrete and distributed time-varying delays, but not necessarily differentiable. The proposed conditions allow us to design the state feedback controllers which stabilize the closed-loop system. By constructing an appropriate Lyapunov-Krasovskii functional, new delay-dependent sufficient conditions for the existence of guaranteed cost control are given in terms of linear matrix inequalities (LMIs). Moreover, we design new quadratic cost functions and minimize their upper bound. Finally, numerical examples are given to illustrate the effectiveness and improvement over some existing results in the literature.
\end{abstract}

Keywords: Guaranteed cost control; Exponential stability; Mixed time-varying delays; Feedback control

\section{Introduction}

In fact, nonlinear systems for modeling the behavior of many engineering systems, such as offshore platforms, earthquake dynamics, electronic circuits, and so on have been widely explored. In some practical nonlinear systems, there are many kinds of nonlinearities, and it is quite difficult to handle all nonlinearities in a single unified framework. Therefore, specialized state observers are designed for different nonlinearities in nonlinear systems [1-7]. Time-delay naturally appears in most real world systems, for instance, aircraft stabilization, ship stabilization, chemical engineering systems, nuclear reactor, population dynamic model, neural network, biological modeling, electrical networks and systems with lossless transmission lines. It is well known that the existence of time-delay may be the cause for instability and poor performance of the system. Therefore, the problem of stability of time-delay systems has thus received considerable attention during the past several decades [8-14].

Recently, the basic theoretical and practical problem of exponential stability and control of a delayed nonlinear system has also been investigated by some researchers [15-18] because it is also an important index to obtain the convergence rates of prescribed time-delay systems. In [15], the problem of exponential stabilization of nonlinear dynamical systems with delayed perturbations was studied by constructing a quadratic Lyapunov functional.

(c) The Author(s) 2018. This article is distributed under the terms of the Creative Commons Attribution 4.0 International License (http://creativecommons.org/licenses/by/4.0/), which permits unrestricted use, distribution, and reproduction in any medium, provided you give appropriate credit to the original author(s) and the source, provide a link to the Creative Commons license, and indicate if changes were made. 
Dong et al. [16] investigated exponential stabilization problem for a class of uncertain nonlinear systems with time delay. Based on the Lyapunov-Krasovskii functional approach, sufficient conditions for exponential stabilization of the system have been presented in terms of a set of matrix inequalities. In [17], the problem of exponential stabilization of nonlinear systems with interval time-varying delays was studied via feedback control. Unfortunately, there have been few papers so far related to the topic of exponential stabilization criteria of a the nonlinear system with interval and distributed time-varying delays via hybrid feedback control. This exponential stabilization of a nonlinear system remains an open problem and it has to be investigated more.

On the other hand, many practical applications of nonlinear systems demand the design of controllers that not only stabilize the system but also minimize a target function, namely the cost function. Thus, the guaranteed cost control technique is an important and useful tool to design stabilizing controllers that guarantee a specified value of the closed-loop cost function. For guaranteed cost control, many valuable results about this topic have been reported for linear systems [19-23], nonlinear systems [17, 24-27], neural network [28-31], complex dynamical network [32-35], and other systems. In [23], the dynamic output feedback guaranteed cost control for linear systems with interval timevarying delays in both states and outputs was investigated. The proposed controller applied only the delayed output measurement to stabilize the closed-loop system and guarantee an adequate level of system performance. On the other hand, a linear-quadratic cost function is studied as a performance measure for the closed-loop system. An optimal cost controller for a linear system with mixed time-varying delays in state and control has been considered in [22]. Moreover, the problem of robust finite-time stabilization with guaranteed cost control for a class of delayed neural networks is considered in [29] with nonlinear quadratic cost functions as a delay performance measure for the closed-loop system. In [17], by applying nonlinear quadratic cost functions, the author studied state feedback guaranteed cost control of nonlinear systems with time-varying delay. So far, unfortunately, there have been no papers related to the topic of guaranteed cost control for an exponentially stable nonlinear system with nonlinear quadratic cost functions including both discrete and distributed delays as their performance measure, simultaneously. Therefore, it is challenging to solve this guaranteed cost problem for nonlinear system with mixed time-delays.

Based on the above discussions, we shall handle the new guaranteed cost control for exponential stability of nonlinear systems with mixed time-delays. The main contributions of this paper are given as follows:

- Nonlinear quadratic cost functions are considered, including both discrete and distributed delays as their performance measure for a closed-loop system. And

$$
\begin{aligned}
J= & \int_{0}^{\infty}\left[x^{T}(t) Z_{1} x(t)+x^{T}\left(t-h_{1}(t)\right) Z_{2} x\left(t-h_{1}(t)\right)+u^{T}(t) Y_{1} u(t)\right. \\
& \left.+\left(\int_{t-d_{1}(t)}^{t} x^{T}(s) d s\right) Z_{3}\left(\int_{t-d_{1}(t)}^{t} x(s) d s\right)\right] d t
\end{aligned}
$$

is first proposed to analyze the problem of guaranteed cost control for a class of nonlinear systems with mixed time-varying delays, which is different from

$[17,22,23,29,31]$. 
- The mixed time-varying delays in state and control input, which consist of discrete and distributed delays, are considered without assuming the differentiability of the time-varying delays.

- The upper bound of given quadratic cost functions is minimized by guaranteed cost control technique.

The proposed conditions allow us to design the state feedback controllers which stabilize the closed-loop system. By constructing an appropriate Lyapunov-Krasovskii functional, new delay-dependent sufficient conditions for the existence of guaranteed cost control are given in terms of linear matrix inequalities (LMIs). Finally, numerical examples are given to illustrate the effectiveness and improvement over some existing results in the literature.

The outline of the paper is organized as follows. Section 2 presents the definitions and some well-known technical propositions for the proof of the main result. Sufficient conditions for the existence of guaranteed cost feedback control for exponential stability of nonlinear system with mixed time-varying delays and numerical examples for showing the effectiveness of the result are presented in Sects. 3-4. The paper ends with conclusions and cited references.

\section{Model description and mathematic preliminaries}

Notations The following notations will be used in this paper: $\mathbb{R}^{+}$denotes the set of all real nonnegative numbers; $\mathbb{R}^{n}$ denotes the $n$-dimensional space and $\|\cdot\|$ denotes the Euclidean vector norm; $A^{n \times m}$ denotes the space of all $n \times m$ matrices; $A^{T}$ denotes the transpose of matrix $A ; A$ is symmetric if $A=A^{T} ; I$ denotes the identity matrix; $\lambda(A)$ denotes the set of all eigenvalues of $A ; \lambda_{\min }(A)=\min \{\operatorname{Re} \lambda ; \lambda \in \lambda(A)\} ; \lambda_{\max }(A)=\max \{\operatorname{Re} \lambda ; \lambda \in \lambda(A)\}$; $x_{t}=\{x(t+s): s \in[-h, 0]\} ;\left\|x_{t}\right\|=\sup _{s \in[-h, 0]}\|x(t+s)\| ; C\left([0, t], \mathbb{R}^{n}\right)$ denotes the set of all $\mathbb{R}^{n}$ valued continuous functions on $[0, t] ; L_{2}\left([0, t], \mathbb{R}^{m}\right)$ denotes the set of all $\mathbb{R}^{m}$-valued square integrable functions on $[0, t]$.

Matrix $A$ is called positive definite $(A>0)$ if $x^{T} A x>0$ for all $x \in \mathbb{R}^{n}, x \neq 0$; matrix $A$ is called semi-positive definite $(A \geq 0)$ if $x^{T} A x \geq 0$ for all $x \in \mathbb{R}^{n} ; A>B$ means $A-B>0$; the symmetric term in a matrix is denoted by $*$.

Consider the nonlinear system with mixed time-varying delays as follows:

$$
\begin{aligned}
\dot{x}(t)= & A x(t)+B x\left(t-h_{1}(t)\right)+C \int_{t-d_{1}(t)}^{t} x(s) d s \\
& +f\left(t, x(t), x\left(t-h_{1}(t)\right), \int_{t-d_{1}(t)}^{t} x(s) d s, u(t)\right)+U(t), \\
U(t)= & D_{1} u(t)+D_{2} u\left(t-h_{2}(t)\right)+D_{3} \int_{t-d_{2}(t)}^{t} u(s) d s, \\
x(t)= & \phi(t), \quad t \in\left[-d_{3}, 0\right], \quad d_{3}=\max \left\{h_{1 \mathrm{M}}, h_{2}, d_{1}, d_{2}\right\},
\end{aligned}
$$

where $x(t) \in \mathbb{R}^{n}, u(t) \in \mathbb{R}^{m}$ are the state and control, respectively, the control $u(\cdot) \in$ $L_{2}\left([0, t], \mathbb{R}^{m}\right), u(t)=K x(t), K$ is a constant matrix gain; $\phi(t) \in C\left(\left[-d_{3}, 0\right], \mathbb{R}^{n}\right)$ is the initial function with the norm

$$
\|\phi\|=\sup _{t \in\left[-d_{3}, 0\right]} \sqrt{\|\phi(t)\|^{2}+\|\dot{\phi}(t)\|^{2}},
$$


while $A, B, C, D_{1}, D_{2}, D_{3}$ are given constant matrices with appropriate dimensions. The delay functions $h_{1}(t), h_{2}(t), d_{1}(t)$ and $d_{2}(t)$ satisfy the conditions:

$$
\begin{aligned}
& 0 \leq h_{1 \mathrm{~m}} \leq h_{1}(t) \leq h_{1 \mathrm{M}}, \quad 0 \leq h_{2}(t) \leq h_{2}, \\
& 0 \leq d_{1}(t) \leq d_{1}, \quad 0 \leq d_{2}(t) \leq d_{2}, \quad h_{12}=h_{1 \mathrm{M}}-h_{1 \mathrm{~m}},
\end{aligned}
$$

where $h_{2}(t)$ and $d_{2}(t)$ are given functions; $f\left(t, x(t), x\left(t-h_{1}(t)\right), \int_{t-d_{1}(t)}^{t} x(s) d s, u(t)\right): \mathbb{R}^{+} \times$ $\mathbb{R}^{n} \times \mathbb{R}^{n} \times \mathbb{R}^{n} \times \mathbb{R}^{m} \rightarrow \mathbb{R}^{n}$ is a given continuous function satisfying $f(t, 0,0,0,0)=0, \forall t \in$ $\mathbb{R}^{+}$, and $f(t, x, y, z, u)$ is globally Lipschitz with respect to $(x, y, z, u)$, such that

$$
\begin{gathered}
\exists a, b, c, d>0: \quad\|f(t, x, y, z, u)\| \leq a\|x\|+b\|y\|+c\|z\|+d\|u\|, \\
\forall(t, x, y, z, u) \in \mathbb{R}^{+} \times \mathbb{R}^{n} \times \mathbb{R}^{n} \times \mathbb{R}^{n} \times \mathbb{R}^{m} .
\end{gathered}
$$

Moreover, throughout this paper, we define the following new nonlinear quadratic cost function of the associated system (1) as follows:

$$
\begin{aligned}
J= & \int_{0}^{\infty}\left[x^{T}(t) Z_{1} x(t)+x^{T}\left(t-h_{1}(t)\right) Z_{2} x\left(t-h_{1}(t)\right)+u^{T}(t) Y_{1} u(t)\right. \\
& \left.+\left(\int_{t-d_{1}(t)}^{t} x^{T}(s) d s\right) Z_{3}\left(\int_{t-d_{1}(t)}^{t} x(s) d s\right)\right] d t
\end{aligned}
$$

where $Z_{1}, Z_{2}, Z_{3} \in \mathbb{R}^{n \times n}$ and $Y_{1} \in \mathbb{R}^{m \times m}$ are positive definite matrices.

Remark 1 This is the first time such a new nonlinear quadratic cost function is defined. If $Z_{3}=0$, the quadratic cost function (3) turns into the quadratic cost function of Niamsup and Phat [17], and if $Z_{2}=0$ and $Z_{3}=0$, the quadratic cost function (3) turns into the quadratic cost function of Thuan and Phat [22]. We can see that the quadratic cost functions of earlier works are included in our work, which can be regarded as a special case of the quadratic cost function.

The objectives of this paper are to design a feedback controller $u(t)=K x(t)$ and a finite number $J^{*}>0$, such that the result of the closed-loop system

$$
\begin{aligned}
\dot{x}(t)= & \left(A+D_{1} K\right) x(t)+B x\left(t-h_{1}(t)\right)+C \int_{t-d_{1}(t)}^{t} x(s) d s \\
& +f\left(t, x(t), x\left(t-h_{1}(t)\right), \int_{t-d_{1}(t)}^{t} x(s) d s, K x(t)\right) \\
& +D_{2} u\left(t-h_{2}(t)\right)+D_{3} \int_{t-d_{2}(t)}^{t} u(s) d s
\end{aligned}
$$

is exponentially stable and the value $J(u) \leq J^{*}$.

To get the main result in this paper, the definitions of exponential stability are introduced as follows. 
Definition 2.1 ([3]) Consider an $\alpha>0$. The zero solution of closed-loop system (4) is $\alpha$ exponentially stabilizable if there exists a positive number $N>0$ such that every solution $x(t, \phi)$ satisfies the following condition

$$
\|x(t, \phi)\| \leq N e^{-\alpha t}\|\phi\|, \quad \forall t \in \mathbb{R}^{+} .
$$

The guaranteed cost control problem to be addressed in this section is formulated as follows.

Definition 2.2 Consider the control system (1). If there exist a continuous stabilizing state feedback control law $u^{*}(t)=K x(t)$ and a positive number $J^{*}$ such that the zero solution of the closed-loop system (4) is exponentially stable and the value (3) satisfies $J\left(u^{*}\right) \leq J^{*}$ then the cost value $J^{*}$ is a guaranteed cost value, $u^{*}(t)$ is a guaranteed cost controller of the system.

Next, we present some preliminary propositions, which are needed in the proof of our main results.

Proposition 2.3 ([8], Cauchy inequality) For any symmetric positive definite matrix $N \in$ $M^{n \times n}$ and $x, y \in \mathbb{R}^{n}$ we have

$$
\pm 2 x^{T} y \leq x^{T} N x+y^{T} N^{-1} y .
$$

Proposition 2.4 ([8], Schur complement lemma) Given constant symmetric matrices $X, Y$ and $Z$ with appropriate dimensions satisfying $X=X^{T}, Y=Y^{T}>0$, one has $X+Z^{T} Y^{-1} Z<0$ if and only if

$$
\left[\begin{array}{cc}
X & Z^{T} \\
* & -Y
\end{array}\right]<0 \quad \text { or } \quad\left[\begin{array}{cc}
-Y & Z \\
* & X
\end{array}\right]<0
$$

Proposition 2.5 ([12]) For any constant matrix $Z=Z^{T}>0$ and positive numbers $h, \bar{h}$ such that the following integrals are well defined, one has

$$
\begin{gathered}
\text { (i) }-\int_{t-h}^{t} x(s)^{T} Z x(s) d s \leq-\frac{1}{h}\left(\int_{t-h}^{t} x(s) d s\right)^{T} Z\left(\int_{t-h}^{t} x(s) d s\right), \\
\text { (ii) }-\int_{-\bar{h}}^{-h} \int_{t+s}^{t} x(\tau)^{T} Z x(\tau) d \tau d s \leq \\
-\frac{2}{\bar{h}^{2}-h^{2}}\left(\int_{-\bar{h}}^{-h} \int_{t+s}^{t} x(\tau) d \tau d s\right)^{T} Z \\
\times\left(\int_{-\bar{h}}^{-h} \int_{t+s}^{t} x(\tau) d \tau d s\right) .
\end{gathered}
$$

Proposition 2.6 ([12]) Let $f_{1}, f_{2}, \ldots, f_{N}: \mathbb{R}^{m} \rightarrow \mathbb{R}$ have positive values in an open subset $D$ of $\mathbb{R}^{m}$. Then, the reciprocally convex combination of $f_{i}$ over $D$ satisfies

$$
\min _{\left\{r_{i} \mid r_{i}>0, \sum_{i} r_{i}=1\right\}} \sum_{i} \frac{1}{r_{i}} f_{i}(t)=\sum_{i} f_{i}(t)+\max _{g_{i j}(t)} \sum_{i \neq j} g_{i, j}(t)
$$


subject to

$$
\left\{g_{i, j}: \mathbb{R}^{m} \rightarrow \mathbb{R}, g_{j, i}(t)=g_{i, j}(t),\left[\begin{array}{cc}
f_{i}(t) & g_{i, j}(t) \\
g_{i, j}(t) & f_{j}(t)
\end{array}\right] \geq 0\right\} .
$$

\section{Guaranteed cost control for exponential stability of a nonlinear system}

In this section, based on Lyapunov-Krasovskii stability theory, the guaranteed cost control of exponential stability for a nonlinear system with mixed time-varying delays is studied. The following theorem presents a sufficient condition for the existence of the guaranteed cost control laws for the exponential stability of the nonlinear system (1).

Theorem 3.1 The nonlinear system (1) with the quadratic cost function (3) is exponentially stabilized with convergence rate $\alpha>0$ if there exist symmetric positive definite matrices $P, Q_{1}, Q_{2}, R_{1}, R_{2}, R_{3}, R_{4}, S_{1}, T_{1}, T_{2}, W_{1}, W_{2}$ and $W_{3}$ satisfying the following $L M I$ :

$$
\Phi=\left[\begin{array}{ccc}
\Phi_{11} & \Phi_{12} & \Phi_{13} \\
* & \Phi_{22} & \Phi_{23} \\
* & * & \Phi_{33}
\end{array}\right]<0
$$

where

$$
\begin{aligned}
& \Phi_{11}=\left[\begin{array}{ccccc}
M_{1,1} & M_{1,2} & M_{1,3} & M_{1,4} & M_{1,5} \\
* & M_{2,2} & 0 & 0 & 0 \\
* & * & M_{3,3} & 0 & 0 \\
* & * & * & M_{4,4} & 0 \\
* & * & * & * & M_{5,5}
\end{array}\right], \\
& \Phi_{12}=\left[\begin{array}{ccccc}
0 & M_{1,7} & M_{1,8} & M_{1,9} & 0 \\
0 & 0 & 0 & 0 & 0 \\
0 & 0 & 0 & 0 & 0 \\
0 & 0 & 0 & 0 & 0 \\
M_{5,6} & M_{5,7} & M_{5,8} & 0 & 0
\end{array}\right] \text {, } \\
& \Phi_{13}=\left[\begin{array}{ccccc}
M_{1,11} & M_{1,12} & M_{1,13} & M_{1,14} & 0 \\
0 & 0 & 0 & 0 & 0 \\
0 & 0 & 0 & 0 & 0 \\
0 & 0 & 0 & 0 & 0 \\
0 & 0 & 0 & M_{5,14} & 0
\end{array}\right] \text {, } \\
& \Phi_{22}=\left[\begin{array}{ccccc}
M_{6,6} & 0 & 0 & 0 & 0 \\
* & M_{7,7} & M_{7,8} & 0 & 0 \\
* & * & M_{8,8} & 0 & 0 \\
* & * & * & M_{9,9} & M_{9,10} \\
* & * & * & * & M_{10,10}
\end{array}\right] \text {, } \\
& \Phi_{23}=\left[\begin{array}{ccccc}
0 & 0 & 0 & 0 & 0 \\
0 & 0 & 0 & 0 & 0 \\
0 & 0 & 0 & 0 & 0 \\
0 & 0 & 0 & M_{9,14} & 0 \\
0 & 0 & 0 & 0 & 0
\end{array}\right],
\end{aligned}
$$




$$
\begin{aligned}
& \Phi_{33}=\left[\begin{array}{ccccc}
M_{11,11} & 0 & 0 & 0 & 0 \\
* & M_{12,12} & 0 & 0 & 0 \\
* & * & M_{13,13} & 0 & 0 \\
* & * & * & M_{14,14} & M_{14,15} \\
* & * & * & * & M_{15,15}
\end{array}\right] \text {, } \\
& M_{1,1}=[A+\alpha I] P+P[A+\alpha I]^{T}+(a+b+c+0.5 d) I-D_{1} D_{1}^{T}+d D_{1} D_{1}^{T} \\
& +0.25 D_{1} Y_{1} D_{1}^{T}+Q_{1}+Q_{2}+d_{1}^{2} T_{2}+3 e^{2 \alpha h_{2}} D_{2} S_{1} D_{2}^{T} \\
& +2 e^{2 \alpha d_{2}} D_{3} T_{1} D_{3}^{T}-e^{-2 \alpha h_{1 \mathrm{~m}}} R_{1}-e^{-2 \alpha h_{1 \mathrm{M}}} R_{2}-2 e^{-4 \alpha h_{1 \mathrm{~m}}} W_{1} \\
& -2 e^{-4 \alpha h_{1 \mathrm{M}}} W_{2}-\frac{2 e^{-4 \alpha h_{1 \mathrm{M}}} h_{12}}{\left(h_{1 \mathrm{M}}+h_{1 \mathrm{~m}}\right)} W_{3}, \\
& M_{1,2}=P, \quad M_{1,3}=D_{1}, \quad M_{1,4}=d_{2}^{2} D_{1}, \quad M_{1,5}=B P, \\
& M_{1,7}=e^{-2 \alpha h_{1 \mathrm{~m}}} R_{1}, \quad M_{1,8}=e^{-2 \alpha h_{1 \mathrm{M}}} R_{2}, \quad M_{1,9}=C P, \\
& M_{1,11}=\frac{2 e^{-4 \alpha h_{1 \mathrm{~m}}}}{h_{1 \mathrm{~m}}} W_{1}, \quad M_{1,12}=\frac{2 e^{-4 \alpha h_{1 \mathrm{M}}}}{h_{1 \mathrm{M}}} W_{2}, \\
& M_{1,13}=\frac{2 e^{-4 \alpha h_{1 M}}}{\left(h_{1 \mathrm{M}}+h_{1 \mathrm{~m}}\right)} W_{3}, \quad M_{1,14}=P A^{T}-0.5 D_{1} D_{1}^{T}, \\
& M_{2,2}=-\left(2 a I+Z_{1}\right)^{-1}, \quad M_{3,3}=-2 e^{2 \alpha h_{2}} S_{1}, \quad M_{4,4}=-4 d_{2}^{2} T_{1}, \\
& M_{5,5}=-2 e^{-2 \alpha h_{1 \mathrm{M}}} R_{3}+e^{-2 \alpha h_{1 \mathrm{M}}}\left(R_{4}+R_{4}^{T}\right), \quad M_{5,6}=P, \\
& M_{5,7}=e^{-2 \alpha h_{1 \mathrm{M}}} R_{3}-e^{-2 \alpha h_{1 \mathrm{M}}} R_{4}, \\
& M_{5,8}=e^{-2 \alpha h_{1 \mathrm{M}}} R_{3}-e^{-2 \alpha h_{1 \mathrm{M}}} R_{4}^{T}, \\
& M_{5,14}=P B^{T}, \quad M_{6,6}=-\left(2 b I+Z_{2}\right)^{-1}, \\
& M_{7,7}=-e^{-2 \alpha h_{1 \mathrm{~m}}} Q_{1}-e^{-2 \alpha h_{1 \mathrm{~m}}} R_{1}-e^{-2 \alpha h_{1 \mathrm{M}}} R_{3}, \\
& M_{7,8}=e^{-2 \alpha h_{1 \mathrm{M}}} R_{4}^{T}, \\
& M_{8,8}=-e^{-2 \alpha h_{1 \mathrm{M}}} Q_{2}-e^{-2 \alpha h_{1 \mathrm{M}}} R_{2}-e^{-2 \alpha h_{1 \mathrm{M}}} R_{3}, \\
& M_{9,9}=-e^{-2 \alpha d_{1}} T_{2}, \quad M_{9,10}=P, \quad M_{9,14}=P C^{T}, \\
& M_{10,10}=-\left(2 c I+Z_{3}\right)^{-1}, \quad M_{11,11}=\frac{-2 e^{-4 \alpha h_{1 \mathrm{~m}}}}{h_{1 \mathrm{~m}}^{2}} W_{1}, \\
& M_{12,12}=\frac{-2 e^{-4 \alpha h_{1 \mathrm{M}}}}{h_{1 \mathrm{M}}^{2}} W_{2} \text {, } \\
& M_{13,13}=\frac{-2 e^{-4 \alpha h_{1 \mathrm{M}}}}{\left(h_{1 \mathrm{M}}^{2}-h_{1 \mathrm{~m}}^{2}\right)} W_{3} \text {, } \\
& M_{14,14}=h_{1 \mathrm{~m}}^{2} R_{1}+h_{1 \mathrm{M}}^{2} R_{2}+h_{12}^{2} R_{3}+h_{1 \mathrm{~m}}^{2} W_{1}+h_{1 \mathrm{M}}^{2} W_{2}+h_{12} h_{1 \mathrm{M}} W_{3} \\
& +3 e^{2 \alpha h_{2}} D_{2} S_{1} D_{2}^{T}+2 e^{2 \alpha d_{2}} D_{3} T_{1} D_{3}^{T} \\
& +(a+b+c+0.5 d) I-2 P \\
& M_{14,15}=h_{2}^{2} D_{1}, \quad M_{15,15}=-4 h_{2}^{2} S_{1} .
\end{aligned}
$$


Moreover, the feedback control is

$$
u(t)=-\frac{1}{2} D_{1}^{T} P^{-1} x(t), \quad t \in \mathbb{R}^{+}
$$

and an upper bound of the quadratic cost function (3) is as follows:

$$
J \leq \lambda_{2}\|\phi\|^{2}
$$

The solution $x(t, \phi)$ satisfies

$$
\|x(t, \phi)\| \leq \sqrt{\frac{\lambda_{2}}{\lambda_{1}}} e^{-\alpha t}\|\phi\|, \quad \forall t \in \mathbb{R}^{+},
$$

where

$$
\begin{aligned}
\lambda_{1}= & \lambda_{\min }\left(P^{-1}\right), \\
\lambda_{2}= & \lambda_{\max }\left(P^{-1}\right)+h_{1 \mathrm{~m}} \lambda_{\max }\left(P^{-1} Q_{1} P^{-1}\right)+h_{1 \mathrm{M}} \lambda_{\max }\left(P^{-1} Q_{2} P^{-1}\right) \\
& +h_{1 \mathrm{~m}}^{3} \lambda_{\max }\left(P^{-1} R_{1} P^{-1}\right)+h_{1 \mathrm{M}}^{3} \lambda_{\max }\left(P^{-1} R_{2} P^{-1}\right) \\
& +h_{12}^{3} \lambda_{\max }\left(P^{-1} R_{3} P^{-1}\right)+\frac{1}{4} h_{2}^{3} \lambda_{\max }\left(P^{-1} D_{1} S_{1}^{-1} D_{1}^{T} P^{-1}\right) \\
& +\frac{1}{4} d_{2}^{3} \lambda_{\max }\left(P^{-1} D_{1} T_{1}^{-1} D_{1}^{T} P^{-1}\right)+d_{1}^{3} \lambda_{\max }\left(P^{-1} T_{2} P^{-1}\right) \\
& +h_{1 \mathrm{~m}}^{3} \lambda_{\max }\left(P^{-1} W_{1} P^{-1}\right)+h_{1 \mathrm{M}}^{3} \lambda_{\max }\left(P^{-1} W_{2} P^{-1}\right) \\
& +h_{12} h_{1 \mathrm{M}}^{2} \lambda_{\max }\left(P^{-1} W_{3} P^{-1}\right) .
\end{aligned}
$$

Proof We let $Y=P^{-1}$ and $y(t)=Y x(t)$. Using the feedback control (6), we consider a candidate Lyapunov-Krasovskii functional for the closed-loop system (4):

$$
V\left(t, x_{t}\right)=\sum_{i=1}^{12} V_{i}\left(t, x_{t}\right)
$$

where

$$
\begin{aligned}
& V_{1}\left(t, x_{t}\right)=x^{T}(t) Y x(t) \\
& V_{2}\left(t, x_{t}\right)=\int_{t-h_{1 \mathrm{~m}}}^{t} e^{2 \alpha(s-t)} x^{T}(s) Y Q_{1} Y x(s) d s \\
& V_{3}\left(t, x_{t}\right)=\int_{t-h_{1 \mathrm{M}}}^{t} e^{2 \alpha(s-t)} x^{T}(s) Y Q_{2} Y x(s) d s \\
& V_{4}\left(t, x_{t}\right)=h_{1 \mathrm{~m}} \int_{-h_{1 \mathrm{~m}}}^{0} \int_{t+s}^{t} e^{2 \alpha(\theta-t)} \dot{x}^{T}(\theta) Y R_{1} Y \dot{x}(\theta) d \theta d s, \\
& V_{5}\left(t, x_{t}\right)=h_{1 \mathrm{M}} \int_{-h_{1 \mathrm{M}}}^{0} \int_{t+s}^{t} e^{2 \alpha(\theta-t)} \dot{x}^{T}(\theta) Y R_{2} Y \dot{x}(\theta) d \theta d s, \\
& V_{6}\left(t, x_{t}\right)=h_{12} \int_{-h_{1 \mathrm{M}}}^{-h_{1 \mathrm{~m}}} \int_{t+s}^{t} e^{2 \alpha(\theta-t)} \dot{x}^{T}(\theta) Y R_{3} Y \dot{x}(\theta) d \theta d s,
\end{aligned}
$$




$$
\begin{aligned}
& V_{7}\left(t, x_{t}\right)=h_{2} \int_{-h_{2}}^{0} \int_{t+s}^{t} e^{2 \alpha(\theta-t)} \dot{u}^{T}(\theta) S_{1}^{-1} \dot{u}(\theta) d \theta d s, \\
& V_{8}\left(t, x_{t}\right)=d_{2} \int_{-d_{2}}^{0} \int_{t+s}^{t} e^{2 \alpha(\theta-t)} u^{T}(\theta) T_{1}^{-1} u(\theta) d \theta d s, \\
& V_{9}\left(t, x_{t}\right)=d_{1} \int_{-d_{1}}^{0} \int_{t+s}^{t} e^{2 \alpha(\theta-t)} x^{T}(\theta) Y T_{2} Y x(\theta) d \theta d s, \\
& V_{10}\left(t, x_{t}\right)=\int_{-h_{1 \mathrm{~m}}}^{0} \int_{\tau}^{0} \int_{t+s}^{t} e^{2 \alpha(\theta+s-t)} \dot{x}^{T}(\theta) Y W_{1} Y \dot{x}(\theta) d \theta d s d \tau, \\
& V_{11}\left(t, x_{t}\right)=\int_{-h_{1 \mathrm{M}}}^{0} \int_{\tau}^{0} \int_{t+s}^{t} e^{2 \alpha(\theta+s-t)} \dot{x}^{T}(\theta) Y W_{2} Y \dot{x}(\theta) d \theta d s d \tau, \\
& V_{12}\left(t, x_{t}\right)=\int_{-h_{1 \mathrm{M}}}^{-h_{1 \mathrm{~m}}} \int_{\tau}^{0} \int_{t+s}^{t} e^{2 \alpha(\theta+s-t)} \dot{x}^{T}(\theta) Y W_{3} Y \dot{x}(\theta) d \theta d s d \tau .
\end{aligned}
$$

It is easy to check that

$$
\lambda_{1}\|x(t)\|^{2} \leq V\left(t, x_{t}\right) \leq \lambda_{2}\left\|x_{t}\right\|^{2}, \quad \forall t \geq 0 .
$$

Taking the derivative of $V_{i}\left(t, x_{t}\right)$ along the solution of the system, we get

$$
\begin{aligned}
\dot{V}_{1}\left(t, x_{t}\right)= & 2 x^{T}(t) Y \dot{x}(t) \\
= & y^{T}(t)\left[A P+P A^{T}\right] y(t)-y^{T}(t) D_{1} D_{1}^{T} y(t)+2 y^{T}(t) B P y\left(t-h_{1}(t)\right) \\
& +2 y^{T}(t) C P \int_{t-d_{1}(t)}^{t} y(s) d s+2 y^{T}(t) f\left(t, x(t), x\left(t-h_{1}(t)\right),\right. \\
& \left.\int_{t-d_{1}(t)}^{t} x(s) d s, u(t)\right)+2 y^{T}(t) D_{2} u\left(t-h_{2}(t)\right) \\
& +2 y^{T}(t) D_{3} \int_{t-d_{2}(t)}^{t} u(s) d s, \\
\dot{V}_{2}\left(t, x_{t}\right)= & y^{T}(t) Q_{1} y(t)-e^{-2 \alpha h_{1 \mathrm{~m}}} y^{T}\left(t-h_{1 \mathrm{~m}}\right) Q_{1} y\left(t-h_{1 \mathrm{~m}}\right)-2 \alpha V_{2}\left(t, x_{t}\right), \\
\dot{V}_{3}\left(t, x_{t}\right)= & y^{T}(t) Q_{2} y(t)-e^{-2 \alpha h_{1 \mathrm{M}}} y^{T}\left(t-h_{1 \mathrm{M}}\right) Q_{2} y\left(t-h_{1 \mathrm{M}}\right)-2 \alpha V_{3}\left(t, x_{t}\right), \\
\dot{V}_{4}\left(t, x_{t}\right) \leq & h_{1 \mathrm{~m}}^{2} \dot{y}^{T}(t) R_{1} \dot{y}(t)-h_{1 \mathrm{~m}} e^{-2 \alpha h_{1 \mathrm{~m}}} \int_{t-h_{1 \mathrm{~m}}}^{t} \dot{y}^{T}(s) R_{1} \dot{y}(s) d s \\
& -2 \alpha V_{4}\left(t, x_{t}\right), \\
\dot{V}_{5}\left(t, x_{t}\right) \leq & h_{1 \mathrm{M}}^{2} \dot{y}^{T}(t) R_{2} \dot{y}(t)-h_{1 \mathrm{M}} e^{-2 \alpha h_{1 \mathrm{M}}} \int_{t-h_{1 \mathrm{M}}}^{t} \dot{y}^{T}(s) R_{2} \dot{y}(s) d s \\
& -2 \alpha V_{5}\left(t, x_{t}\right), \\
\dot{V}_{7}\left(t, x_{t}\right) \leq & h_{2}^{2} \dot{u}^{T}(t) S_{1}^{-1} \dot{u}(t)-h_{2} e^{-2 \alpha h_{2}} \int_{t-h_{2}}^{t} \dot{u}^{T}(s) S_{1}^{-1} \dot{u}(s) d s \\
& -2 \alpha V_{6}\left(t, x_{t}\right), \\
\dot{V}_{6}\left(t, x_{t}\right) \leq & h_{12}^{2} \dot{y}^{T}(t) R_{3} \dot{y}(t)-h_{12} e^{-2 \alpha h_{1 \mathrm{M}}} \int_{t-h_{1 \mathrm{M}}}^{t-h_{1 \mathrm{~m}}} \dot{y}^{T}(s) R_{3} \dot{y}(s) d s \\
&
\end{aligned}
$$




$$
\begin{aligned}
& -2 \alpha V_{7}\left(t, x_{t}\right), \\
\dot{V}_{8}\left(t, x_{t}\right) \leq & d_{2}^{2} u^{T}(t) T_{1}^{-1} u(t)-d_{2} e^{-2 \alpha d_{2}} \int_{t-d_{2}}^{t} u^{T}(s) T_{1}^{-1} u(s) d s \\
& -2 \alpha V_{8}\left(t, x_{t}\right), \\
\dot{V}_{9}\left(t, x_{t}\right) \leq & d_{1}^{2} y^{T}(t) T_{2} y(t)-d_{1} e^{-2 \alpha d_{1}} \int_{t-d_{1}}^{t} y^{T}(s) T_{2} y(s) d s \\
& -2 \alpha V_{9}\left(t, x_{t}\right), \\
\dot{V}_{10}\left(t, x_{t}\right) \leq & h_{1 \mathrm{~m}}^{2} \dot{y}^{T}(t) W_{1} \dot{y}(t)-e^{-4 \alpha h_{1 \mathrm{~m}}} \int_{-h_{1 \mathrm{~m}}}^{0} \int_{t+\tau}^{t} \dot{y}^{T}(s) W_{1} \dot{y}(s) d s d \tau \\
& -2 \alpha V_{10}\left(t, x_{t}\right), \\
\dot{V}_{11}\left(t, x_{t}\right) \leq & h_{1 \mathrm{M}}^{2} \dot{y}^{T}(t) W_{2} \dot{y}(t)-e^{-4 \alpha h_{1 \mathrm{M}}} \int_{-h_{1 \mathrm{M}}}^{0} \int_{t+\tau}^{t} \dot{y}^{T}(s) W_{2} \dot{y}(s) d s d \tau \\
& -2 \alpha V_{11}\left(t, x_{t}\right), \\
& -2 \alpha V_{12}\left(t, x_{t}\right) . \\
\dot{V}_{12}\left(t, x_{t}\right) \leq & h_{12} h_{1 \mathrm{M}} \dot{y}^{T}(t) W_{3} \dot{y}(t)-e^{-4 \alpha h_{1 \mathrm{M}}} \int_{-h_{1 \mathrm{M}}}^{-h_{1 \mathrm{~m}}} \int_{t+\tau}^{t} \dot{y}^{T}(s) W_{3} \dot{y}(s) d s d \tau \\
&
\end{aligned}
$$

We have

$$
\int_{t-h_{1 \mathrm{M}}}^{t-h_{1 \mathrm{~m}}} \dot{y}^{T}(s) R_{3} \dot{y}(s) d s=\int_{t-h_{1 \mathrm{M}}}^{t-h_{1}(t)} \dot{y}^{T}(s) R_{3} \dot{y}(s) d s+\int_{t-h_{1}(t)}^{t-h_{1 \mathrm{~m}}} \dot{y}^{T}(s) R_{3} \dot{y}(s) d s .
$$

Using Proposition 2.5, we have

$$
\begin{aligned}
&-h_{12} e^{-2 \alpha h_{1 \mathrm{M}}} \int_{t-h_{1 \mathrm{M}}}^{t-h_{1}(t)} \dot{y}^{T}(s) R_{3} \dot{y}(s) d s \\
& \leq- \frac{h_{12} e^{-2 \alpha h_{1 \mathrm{M}}}}{h_{1 \mathrm{M}}-h_{1}(t)}\left[y\left(t-h_{1}(t)\right)-y\left(t-h_{1 \mathrm{M}}\right)\right]^{T} R_{3} \\
& \times\left[y\left(t-h_{1}(t)\right)-y\left(t-h_{1 \mathrm{M}}\right)\right] \\
&-\frac{h_{12} e^{-2 \alpha h_{1 \mathrm{M}}}}{h_{1}(t)-h_{1 \mathrm{~m}}}\left[y\left(t-h_{1 \mathrm{~m}}\right)-y\left(t-h_{1}(t)\right)\right]^{T} R_{3} \\
& \times\left[y\left(t-h_{1 \mathrm{~m}}\right)-y\left(t-h_{1}(t)\right)\right] .
\end{aligned}
$$

Given $\left[\begin{array}{ll}R_{3} & R_{4} \\ R_{4}^{T} & R_{3}\end{array}\right] \geq 0$, using Proposition 2.6 with $r_{1}=\left(h_{1 \mathrm{M}}-h_{1}(t)\right) / h_{12}$ and $r_{2}=\left(h_{1}(t)-\right.$ $\left.h_{1 \mathrm{~m}}\right) / h_{12}$ gives the following inequality:

$$
\begin{gathered}
{\left[\begin{array}{c}
\sqrt{\frac{r_{2}}{r_{1}}}\left[y\left(t-h_{1}(t)\right)-y\left(t-h_{1 \mathrm{M}}\right)\right] \\
-\sqrt{\frac{r_{1}}{r_{2}}}\left[y\left(t-h_{1 \mathrm{~m}}\right)-y\left(t-h_{1}(t)\right)\right]
\end{array}\right]^{T}\left[\begin{array}{ll}
R_{3} & R_{4} \\
R_{4}^{T} & R_{3}
\end{array}\right]} \\
\times\left[\begin{array}{c}
\sqrt{\frac{r_{2}}{r_{1}}}\left[y\left(t-h_{1}(t)\right)-y\left(t-h_{1 \mathrm{M}}\right)\right] \\
-\sqrt{\frac{r_{1}}{r_{2}}}\left[y\left(t-h_{1 \mathrm{~m}}\right)-y\left(t-h_{1}(t)\right)\right]
\end{array}\right] \geq 0 .
\end{gathered}
$$


Then

$$
\begin{aligned}
& -\frac{h_{1 \mathrm{M}}-h_{1 \mathrm{~m}}}{h_{1 \mathrm{M}}-h_{1}(t)}\left[y\left(t-h_{1}(t)\right)-y\left(t-h_{1 \mathrm{M}}\right)\right]^{T} R_{3}\left[y\left(t-h_{1}(t)\right)-y\left(t-h_{1 \mathrm{M}}\right)\right] \\
& -\frac{h_{1 \mathrm{M}}-h_{1 \mathrm{~m}}}{h_{1}(t)-h_{1 \mathrm{~m}}}\left[y\left(t-h_{1 \mathrm{~m}}\right)-y\left(t-h_{1}(t)\right)\right]^{T} R_{3}\left[y\left(t-h_{1 \mathrm{~m}}\right)-y\left(t-h_{1}(t)\right)\right] \\
& \leq-\left[y\left(t-h_{1}(t)\right)-y\left(t-h_{1 \mathrm{M}}\right)\right]^{T} R_{3}\left[y\left(t-h_{1}(t)\right)-y\left(t-h_{1 \mathrm{M}}\right)\right] \\
& -\left[y\left(t-h_{1 \mathrm{~m}}\right)-y\left(t-h_{1}(t)\right)\right]^{T} R_{3}\left[y\left(t-h_{1 \mathrm{~m}}\right)-y\left(t-h_{1}(t)\right)\right] \\
& \text { - }\left[y\left(t-h_{1}(t)\right)-y\left(t-h_{1 \mathrm{M}}\right)\right]^{T} R_{4}\left[y\left(t-h_{1 \mathrm{~m}}\right)-y\left(t-h_{1}(t)\right)\right] \\
& -\left[y\left(t-h_{1 \mathrm{~m}}\right)-y\left(t-h_{1}(t)\right)\right]^{T} R_{4}^{T}\left[y\left(t-h_{1}(t)\right)-y\left(t-h_{1 \mathrm{M}}\right)\right] .
\end{aligned}
$$

Therefore, we have

$$
\begin{aligned}
\dot{V}_{4}\left(t, x_{t}\right) \leq & h_{1 \mathrm{~m}}^{2} \dot{y}^{T}(t) R_{1} \dot{y}(t)-2 \alpha V_{4}\left(t, x_{t}\right) \\
& -e^{-2 \alpha h_{1 \mathrm{~m}}}\left[y(t)-y\left(t-h_{1 \mathrm{~m}}\right)\right]^{T} R_{1}\left[y(t)-y\left(t-h_{1 \mathrm{~m}}\right)\right], \\
\dot{V}_{5}\left(t, x_{t}\right) \leq & h_{1 \mathrm{M}}^{2} \dot{y}^{T}(t) R_{2} \dot{y}(t)-2 \alpha V_{5}\left(t, x_{t}\right) \\
& -e^{-2 \alpha h_{1 \mathrm{M}}}\left[y(t)-y\left(t-h_{1 \mathrm{M}}\right)\right]^{T} R_{2}\left[y(t)-y\left(t-h_{1 \mathrm{M}}\right)\right], \\
\dot{V}_{6}\left(t, x_{t}\right) \leq & h_{12}^{2} \dot{y}^{T}(t) R_{3} \dot{y}(t)-2 \alpha V_{6}\left(t, x_{t}\right) \\
& -e^{-2 \alpha h_{1 \mathrm{M}}}\left[y\left(t-h_{1}(t)\right)-y\left(t-h_{1 \mathrm{M}}\right)\right]^{T} R_{3}\left[y\left(t-h_{1}(t)\right)-y\left(t-h_{1 \mathrm{M}}\right)\right] \\
& -e^{-2 \alpha h_{1 \mathrm{M}}}\left[y\left(t-h_{1 \mathrm{~m}}\right)-y\left(t-h_{1}(t)\right)\right]^{T} R_{3}\left[y\left(t-h_{1 \mathrm{~m}}\right)-y\left(t-h_{1}(t)\right)\right] \\
& -e^{-2 \alpha h_{1 \mathrm{M}}}\left[y\left(t-h_{1}(t)\right)-y\left(t-h_{1 \mathrm{M}}\right)\right]^{T} R_{4}\left[y\left(t-h_{1 \mathrm{~m}}\right)-y\left(t-h_{1}(t)\right)\right] \\
& -e^{-2 \alpha h_{1 \mathrm{M}}}\left[y\left(t-h_{1 \mathrm{~m}}\right)-y\left(t-h_{1}(t)\right)\right]^{T} R_{4}^{T}\left[y\left(t-h_{1}(t)\right)-y\left(t-h_{1 \mathrm{M}}\right)\right] .
\end{aligned}
$$

Applying Propositions 2.3, 2.5 and the Newton-Leibniz formula gives

$$
\begin{aligned}
-h_{2} e^{-2 \alpha h_{2}} \int_{t-h_{2}}^{t} \dot{u}^{T}(s) S_{1}^{-1} \dot{u}(s) d s & \\
\leq & -e^{-2 \alpha h_{2}}\left[\int_{t-h_{2}(t)}^{t} \dot{u}(s) d s\right]^{T} S_{1}^{-1}\left[\int_{t-h_{2}(t)}^{t} \dot{u}(s) d s\right] \\
= & -e^{-2 \alpha h_{2}} u^{T}(t) S_{1}^{-1} u(t)+2 e^{-2 \alpha h_{2}} u^{T}(t) S_{1}^{-1} u\left(t-h_{2}(t)\right) \\
& -e^{-2 \alpha h_{2}} u^{T}\left(t-h_{2}(t)\right) S_{1}^{-1} u\left(t-h_{2}(t)\right) \\
\leq & 0.5 e^{-2 \alpha h_{2}} y^{T}(t) D_{1} S_{1}^{-1} D_{1}^{T} y(t) \\
& +\frac{e^{-2 \alpha h_{2}}}{3} u^{T}\left(t-h_{2}(t)\right) S_{1}^{-1} u\left(t-h_{2}(t)\right) \\
& -e^{-2 \alpha h_{2}} u^{T}\left(t-h_{2}(t)\right) S_{1}^{-1} u\left(t-h_{2}(t)\right) \\
-d_{2} e^{-2 \alpha d_{2}} \int_{t-d_{2}}^{t} u^{T}(s) T_{1}^{-1} u(s) d s & \\
\leq & -d_{2}(t) e^{-2 \alpha d_{2}} \int_{t-d_{2}(t)}^{t} u^{T}(s) T_{1}^{-1} u(s) d s
\end{aligned}
$$


and

$$
\begin{aligned}
& -d_{1} e^{-2 \alpha d_{1}} \int_{t-d_{1}}^{t} y^{T}(s) T_{2} y(s) d s \\
& \leq-e^{-2 \alpha d_{1}}\left[\int_{t-d_{1}(t)}^{t} y^{T}(s) d s\right] T_{2}\left[\int_{t-d_{1}(t)}^{t} y(s) d s\right] .
\end{aligned}
$$

Using Proposition 2.5, we have

$$
\begin{aligned}
& -e^{-4 \alpha h_{1 \mathrm{~m}}} \int_{-h_{1 \mathrm{~m}}}^{0} \int_{t+\tau}^{t} \dot{y}^{T}(s) W_{1} \dot{y}(s) d s d \tau \\
& \leq \frac{-2 e^{-4 \alpha h_{1 \mathrm{~m}}}}{h_{1 \mathrm{~m}}^{2}}\left[h_{1 \mathrm{~m}} y(t)-\int_{t-h_{1 \mathrm{~m}}}^{t} y(\tau) d \tau\right]^{T} W_{1}\left[h_{1 \mathrm{~m}} y(t)-\int_{t-h_{1 \mathrm{~m}}}^{t} y(\tau) d \tau\right], \\
& -e^{-4 \alpha h_{1 \mathrm{M}}} \int_{-h_{1 \mathrm{M}}}^{0} \int_{t+\tau}^{t} \dot{y}^{T}(s) W_{2} \dot{y}(s) d s d \tau \\
& \leq \frac{-2 e^{-4 \alpha h_{1 \mathrm{M}}}}{h_{1 \mathrm{M}}^{2}}\left[h_{1 \mathrm{M}} y(t)-\int_{t-h_{1 \mathrm{M}}}^{t} y(\tau) d \tau\right]^{T} W_{2}\left[h_{1 \mathrm{M}} y(t)-\int_{t-h_{1 \mathrm{M}}}^{t} y(\tau) d \tau\right]
\end{aligned}
$$

and

$$
\begin{aligned}
& -e^{-4 \alpha h_{1 \mathrm{M}}} \int_{-h_{1 \mathrm{M}}}^{-h_{1 \mathrm{~m}}} \int_{t+\tau}^{t} \dot{y}^{T}(s) W_{3} \dot{y}(s) d s d \tau \\
& \leq \frac{-2 e^{-4 \alpha h_{1 \mathrm{M}}}}{h_{1 \mathrm{M}}^{2}-h_{1 \mathrm{~m}}^{2}}\left[h_{12} y(t)-\int_{t-h_{1 \mathrm{M}}}^{t-h_{1 \mathrm{~m}}} y(\tau) d \tau\right]^{T} W_{3}\left[h_{12} y(t)-\int_{t-h_{1 \mathrm{M}}}^{t-h_{1 \mathrm{~m}}} y(\tau) d \tau\right] .
\end{aligned}
$$

Using Proposition 2.3, we get

$$
\begin{aligned}
2 y^{T}(t) D_{2} u\left(t-h_{2}(t)\right) \leq & 3 e^{2 \alpha h_{2}} y^{T}(t) D_{2} S_{1} D_{2}^{T} y(t) \\
& +\frac{e^{-2 \alpha h_{2}}}{3} u^{T}\left(t-h_{2}(t)\right) S_{1}^{-1} u\left(t-h_{2}(t)\right), \\
2 y^{T}(t) D_{3} \int_{t-d_{2}(t)}^{t} u(s) d s \leq & 2 e^{2 \alpha d_{2}} y^{T}(t) D_{3} T_{1} D_{3}^{T} y(t) \\
\leq & 2 e^{2 \alpha d_{2}} y^{T}(t) D_{3} T_{1} D_{3}^{T} y(t) \\
& +\frac{d_{2}(t) e^{-2 \alpha d_{2}}}{2} \int_{t-d_{2}(t)}^{t} u^{T}(s) T_{1}^{-1} u(s) d s .
\end{aligned}
$$

From (2) and Proposition 2.3, we have

$$
\begin{aligned}
& 2 y^{T}(t) f\left(t, P y(t), P y\left(t-h_{1}(t)\right), P \int_{t-d_{1}(t)}^{t} y(s) d s, u(t)\right) \\
& \leq 2\left(a\|P y(t)\|+b\left\|P y\left(t-h_{1}(t)\right)\right\|+c\left\|P \int_{t-d_{1}(t)}^{t} y(s) d s\right\|+d\|u(t)\|\right)\|y(t)\| \\
& \leq(a+b+c+0.5 d) y^{T}(t) y(t)+a y^{T}(t) P^{2} y(t)+b y^{T}\left(t-h_{1}(t)\right) P^{2} y\left(t-h_{1}(t)\right) \\
& \quad+c \int_{t-d_{1}(t)}^{t} y^{T}(s) d s P^{2} \int_{t-d_{1}(t)}^{t} y(s) d s+0.5 d y^{T}(t) D_{1} D_{1}^{T} y(t) .
\end{aligned}
$$


Now we obtain the following identity:

$$
\begin{aligned}
0= & P \dot{y}(t)-A P y(t)-B P y\left(t-h_{1}(t)\right)-C P \int_{t-d_{1}(t)}^{t} y(s) d s \\
& -f\left(t, x(t), x\left(t-h_{1}(t)\right), \int_{t-d_{1}(t)}^{t} x(s) d s, u(t)\right) \\
& -D_{1} u(t)-D_{2} u\left(t-h_{2}(t)\right)-D_{3} \int_{t-d_{2}(t)}^{t} u(s) d s \\
= & -2 \dot{y}^{T}(t) P \dot{y}(t)+2 \dot{y}^{T}(t) A P y(t)+2 \dot{y}^{T}(t) B P y\left(t-h_{1}(t)\right) \\
& +2 \dot{y}^{T}(t) C P \int_{t-d_{1}(t)}^{t} y(s) d s-\dot{y}^{T}(t) D_{1} D_{1}^{T} y(t) \\
& +2 \dot{y}^{T}(t) f\left(t, x(t), x\left(t-h_{1}(t)\right), \int_{t-d_{1}(t)}^{t} x(s) d s, u(t)\right) \\
& +2 \dot{y}^{T}(t) D_{2} u\left(t-h_{2}(t)\right)+2 \dot{y}^{T}(t) D_{3} \int_{t-d_{2}(t)}^{t} u(s) d s,
\end{aligned}
$$

from which we have

$$
\begin{aligned}
2 \dot{y}^{T}(t) D_{2} u\left(t-h_{2}(t)\right) \leq & 3 e^{2 \alpha h_{2}} \dot{y}^{T}(t) D_{2} S_{1} D_{2}^{T} \dot{y}(t) \\
& +\frac{e^{-2 \alpha h_{2}}}{3} u^{T}\left(t-h_{2}(t)\right) S_{1}^{-1} u\left(t-h_{2}(t)\right), \\
2 \dot{y}^{T}(t) D_{3} \int_{t-d_{2}(t)}^{t} u(s) d s \leq & 2 e^{2 \alpha d_{2}} \dot{y}^{T}(t) D_{3} T_{1} D_{3}^{T} \dot{y}(t) \\
& +\frac{d_{2}(t) e^{-2 \alpha d_{2}}}{2} \int_{t-d_{2}(t)}^{t} u^{T}(s) T_{1}^{-1} u(s) d s,
\end{aligned}
$$

and

$$
\begin{aligned}
& 2 \dot{y}^{T}(t) f\left(t, x(t), x\left(t-h_{1}(t)\right), \int_{t-d_{1}(t)}^{t} x(s) d s, u(t)\right) \\
& \leq(a+b+c+0.5 d) \dot{y}^{T}(t) \dot{y}(t)+a y^{T}(t) P^{2} y(t)+b y^{T}\left(t-h_{1}(t)\right) P^{2} y\left(t-h_{1}(t)\right) \\
& \quad+c\left(\int_{t-d_{1}(t)}^{t} y^{T}(s) d s\right) P^{2}\left(\int_{t-d_{1}(t)}^{t} y(s) d s\right)+0.5 d y^{T}(t) D_{1} D_{1}^{T} y(t) .
\end{aligned}
$$

We let

$$
\begin{aligned}
& L\left(t, x(t), x\left(t-h_{1}(t)\right), \int_{t-d_{1}(t)}^{t} x(s) d s, u(t)\right) \\
& \leq\left[y^{T}(t) P Z_{1} P y(t)+y^{T}\left(t-h_{1}(t)\right) P Z_{2} P y\left(t-h_{1}(t)\right)\right. \\
& \left.\quad+\left(\int_{t-d_{1}(t)}^{t} y^{T}(s) d s\right) P Z_{3} P\left(\int_{t-d_{1}(t)}^{t} y(s) d s\right)\right]+0.25 y^{T}(t) D_{1} Y_{1} D_{1}^{T} y(t) .
\end{aligned}
$$


From (10)-(29), we obtain

$$
\begin{aligned}
\dot{V}\left(t, x_{t}\right)+2 \alpha V\left(t, x_{t}\right) \leq & \xi^{T}(t) \Pi \xi(t) \\
& -L\left(t, x(t), x\left(t-h_{1}(t)\right), \int_{t-d_{1}(t)}^{t} x(s) d s, u(t)\right),
\end{aligned}
$$

where

$$
\begin{aligned}
& \xi(t)=\left[y(t), y\left(t-h_{1}(t)\right), y\left(t-h_{1 \mathrm{~m}}\right), y\left(t-h_{1 \mathrm{M}}\right), \int_{t-d_{1}(t)}^{t} y(s) d s,\right. \\
& \left.\int_{t-h_{1 \mathrm{~m}}}^{t} y(\tau) d \tau, \int_{t-h_{1 \mathrm{M}}}^{t} y(\tau) d \tau, \int_{t-h_{1 \mathrm{M}}}^{t-h_{1 \mathrm{~m}}} y(\tau) d \tau, \dot{y}(t)\right], \\
& \Pi=\left[\begin{array}{ccc}
\Pi_{11} & \Pi_{12} & \Pi_{13} \\
* & \Pi_{22} & \Pi_{23} \\
* & * & \Pi_{33}
\end{array}\right]<0 \\
& \Pi_{11}=\left[\begin{array}{ccc}
N_{1,1} & N_{1,2} & N_{1,3} \\
* & N_{2,2} & N_{2,3} \\
* & * & N_{3,3}
\end{array}\right], \quad \Pi_{12}=\left[\begin{array}{ccc}
N_{1,4} & N_{1,5} & N_{1,6} \\
N_{2,4} & 0 & 0 \\
N_{3,4} & 0 & 0
\end{array}\right] \text {, } \\
& \Pi_{13}=\left[\begin{array}{ccc}
N_{1,7} & N_{1,8} & N_{1,9} \\
0 & 0 & N_{2,9} \\
0 & 0 & 0
\end{array}\right], \quad \Pi_{22}=\left[\begin{array}{ccc}
N_{4,4} & 0 & 0 \\
* & N_{5,5} & 0 \\
* & * & N_{6,6}
\end{array}\right] \text {, } \\
& \Pi_{23}=\left[\begin{array}{ccc}
0 & 0 & 0 \\
0 & 0 & N_{5,9} \\
0 & 0 & 0
\end{array}\right], \quad \Pi_{33}=\left[\begin{array}{ccc}
N_{7,7} & 0 & 0 \\
* & N_{8,8} & 0 \\
* & * & N_{9,9}
\end{array}\right] \text {, } \\
& N_{1,1}=[A+\alpha I] P+P[A+\alpha I]^{T}+(a+b+c+0.5 d) I-D_{1} D_{1}^{T}+d D_{1} D_{1}^{T} \\
& +0.25 D_{1} Y_{1} D_{1}^{T}+Q_{1}+Q_{2}+d_{1}^{2} T_{2}+3 e^{2 \alpha h_{2}} D_{2} S_{1} D_{2}^{T}+2 e^{2 \alpha d_{2}} D_{3} T_{1} D_{3}^{T} \\
& -e^{-2 \alpha h_{1 \mathrm{~m}}} R_{1}-e^{-2 \alpha h_{1 \mathrm{M}}} R_{2}-2 e^{-4 \alpha h_{1 \mathrm{~m}}} W_{1}-2 e^{-4 \alpha h_{1 \mathrm{M}}} W_{2} \\
& -\frac{2 e^{-4 \alpha h_{1 \mathrm{M}}} h_{12}}{\left(h_{1 \mathrm{M}}+h_{1 \mathrm{~m}}\right)} W_{3}+P\left(2 a I+Z_{1}\right) P+0.5 e^{-2 \alpha h_{2}} D_{1} S_{1}^{-1} D_{1}^{T} \\
& +0.25 d_{2}^{2} D_{1} T_{1}^{-1} D_{1}^{T} \\
& N_{1,2}=B P \text {, } \\
& N_{1,3}=e^{-2 \alpha h_{1 \mathrm{~m}}} R_{1}, \quad N_{1,4}=e^{-2 \alpha h_{1 \mathrm{M}}} R_{2}, N_{1,5}=C P, \\
& N_{1,6}=\frac{2 e^{-4 \alpha h_{1 \mathrm{~m}}}}{h_{1 \mathrm{~m}}} W_{1}, \quad N_{1,7}=\frac{2 e^{-4 \alpha h_{1 \mathrm{M}}}}{h_{1 \mathrm{M}}} W_{2}, \\
& N_{1,8}=\frac{2 e^{-4 \alpha h_{1 \mathrm{M}}}}{\left(h_{1 \mathrm{M}}+h_{1 \mathrm{~m}}\right)} W_{3}, \quad N_{1,9}=P A^{T}-0.5 D_{1} D_{1}^{T}, \\
& N_{2,2}=-2 e^{-2 \alpha h_{1 \mathrm{M}}} R_{3}+e^{-2 \alpha h_{1 \mathrm{M}}}\left(R_{4}+R_{4}^{T}\right)+P\left(2 b I+Z_{2}\right) P \text {, } \\
& N_{2,3}=e^{-2 \alpha h_{1 \mathrm{M}}} R_{3}-e^{-2 \alpha h_{1 \mathrm{M}}} R_{4}, \\
& N_{2,4}=e^{-2 \alpha h_{1 \mathrm{M}}} R_{3}-e^{-2 \alpha h_{1 \mathrm{M}}} R_{4}^{T},
\end{aligned}
$$




$$
\begin{aligned}
N_{2,9}= & P B^{T}, \\
N_{3,3}= & -e^{-2 \alpha h_{1 \mathrm{~m}}} Q_{1}-e^{-2 \alpha h_{1 \mathrm{~m}}} R_{1}-e^{-2 \alpha h_{1 \mathrm{M}}} R_{3}, \\
N_{3,4}= & e^{-2 \alpha h_{1 \mathrm{M}}} R_{4}^{T}, \\
N_{4,4}= & -e^{-2 \alpha h_{1 \mathrm{M}}} Q_{2}-e^{-2 \alpha h_{1 \mathrm{M}}} R_{2}-e^{-2 \alpha h_{1 \mathrm{M}}} R_{3}, \\
N_{5,5}= & -e^{-2 \alpha d_{1}} T_{2}+P\left(2 c I+Z_{3}\right) P, \quad N_{5,9}=P C^{T}, \\
N_{6,6}= & \frac{-2 e^{-4 \alpha h_{1 \mathrm{~m}}}}{h_{1 \mathrm{~m}}^{2}} W_{1}, \quad N_{7,7}=\frac{-2 e^{-4 \alpha h_{1 \mathrm{M}}}}{h_{1 \mathrm{M}}^{2}} W_{2}, \\
N_{8,8}= & \frac{-2 e^{-4 \alpha h_{1 \mathrm{M}}}}{\left(h_{1 \mathrm{M}}^{2}-h_{1 \mathrm{~m}}^{2}\right)} W_{3}, \\
N_{9,9}= & h_{1 \mathrm{~m}}^{2} R_{1}+h_{1 \mathrm{M}}^{2} R_{2}+h_{12}^{2} R_{3}+h_{1 \mathrm{~m}}^{2} W_{1}+h_{1 \mathrm{M}}^{2} W_{2}+h_{12} h_{1 \mathrm{M}} W_{3} \\
& +3 e^{2 \alpha h_{2}} D_{2} S_{1} D_{2}^{T}+2 e^{2 \alpha d_{2}} D_{3} T_{1} D_{3}^{T}+(a+b+c+0.5 d) I-2 P \\
& +0.25 h_{2}^{2} D_{1} S_{1}^{-1} D_{1}^{T} .
\end{aligned}
$$

Using Proposition 2.4 (Schur complement lemma), condition (5) is equivalent to condition $\Pi<0$. Thus, from (5)-(29) and (30), we obtain

$$
\dot{V}\left(t, x_{t}\right)+2 \alpha V\left(t, x_{t}\right) \leq L\left(t, x(t), x\left(t-h_{1}(t)\right), \int_{t-d_{1}(t)}^{t} x(s) d s, u(t)\right)
$$

Since $L\left(t, x(t), x\left(t-h_{1}(t)\right), \int_{t-d_{1}(t)}^{t} x(s) d s, u(t)\right)>0$, we have

$$
\dot{V}\left(t, x_{t}\right) \leq-2 \alpha V\left(t, x_{t}\right), \quad \forall t \in \mathbb{R}^{+} .
$$

Integrating both sides of (32) from 0 to $t$, we obtain

$$
V\left(t, x_{t}\right) \leq V\left(0, x_{0}\right) e^{-2 \alpha t}, \quad \forall t \in \mathbb{R}^{+} .
$$

Furthermore, taking condition (9) into account in (33), we have

$$
\lambda_{1}\|x(t, \phi)\|^{2} \leq V\left(t, x_{t}\right) \leq V\left(0, x_{0}\right) e^{-2 \alpha t} \leq \lambda_{2} e^{-2 \alpha t}\|\phi\|^{2},
$$

and then

$$
\|x(t, \phi)\| \leq \sqrt{\frac{\lambda_{2}}{\lambda_{1}}} e^{-\alpha t}\|\phi\|, \quad \forall t \geq 0
$$

which implies the exponential stability of the closed-loop system (4).

To find an upper bound of the cost function (3), we consider the derived condition (31) and, since $V\left(t, x_{t}\right)>0$, we have

$$
\dot{V}\left(t, x_{t}\right) \leq-L\left(t, x(t), x\left(t-h_{1}(t)\right), \int_{t-d_{1}(t)}^{t} x(s) d s, u(t)\right), \quad \forall t \in \mathbb{R}^{+}
$$


Integrating both sides of (34) from 0 to $t$, we obtain

$$
\int_{0}^{t} L\left(t, x(t), x\left(t-h_{1}(t)\right), \int_{t-d_{1}(t)}^{t} x(s) d s, u(t)\right) d t \leq V\left(0, x_{0}\right)-V\left(t, x_{t}\right) \leq V\left(0, x_{0}\right),
$$

because $V\left(t, x_{t}\right)>0$. Hence, letting $t \rightarrow \infty$, we finally obtain

$$
J=\int_{0}^{\infty} L\left(t, x(t), x\left(t-h_{1}(t)\right), \int_{t-d_{1}(t)}^{t} x(s) d s, u(t)\right) d t \leq V\left(0, x_{0}\right) \leq \lambda_{2}\|\phi\|^{2}=J^{*} .
$$

This completes the proof of the theorem.

Remark 2 In the proof of Theorem 3.1, we need to cancel some integral terms in equation (10). In order to do that, we need Proposition 2.3 to estimate some integral terms. This allows achieving such a goal but using this technique causes the complexity of the presented conditions.

Remark 3 In addition, when $C=0, D_{2}=0$ and $D_{3}=0$, system (1) reduces to a nonlinear system with interval time-varying delay of the form

$$
\begin{aligned}
& \dot{x}(t)=A x(t)+B x\left(t-h_{1}(t)\right)+D_{1} u(t)+f\left(t, x(t), x\left(t-h_{1}(t)\right), u(t)\right), \\
& x(t)=\phi(t), \quad t \in\left[-h_{1 \mathrm{M}}, 0\right],
\end{aligned}
$$

which was proposed by Niamsup and Phat [17]. If $f(\cdot)=0$, then system (1) turns into the linear system with mixed interval time-varying delays considered by Thuan and Phat [22]. Hence, the previous systems were included in the nonlinear system (1), which can be regarded as a special case of a nonlinear system. According to the proof technique in Theorem 3.1, the guaranteed cost control of exponential stability of a nonlinear system with interval time-varying delay (35) can be easily obtained. Thus, we get the following result from Theorem 3.1.

Corollary 3.2 Consider the system (1) and cost function (3) for given $\alpha>0, C=0, D_{2}=0$ and $D_{3}=0$. If there exist symmetric positive definite matrices $P, Q_{1}, Q_{2}, R_{1}, R_{2}, R_{3}, R_{4}, W_{1}$, $W_{2}$ and $W_{3}$ satisfying the LMI

$$
\Omega=\left[\begin{array}{cc}
\Omega_{11} & \Omega_{12} \\
* & \Omega_{22}
\end{array}\right]<0,
$$

then

$$
u(t)=-\frac{1}{2} D_{1}^{T} P^{-1} x(t), \quad J^{*}=\lambda_{2}\|\phi\|^{2}
$$

are the guaranteed cost controller and guaranteed cost value, respectively, where

$$
\Omega_{11}=\left[\begin{array}{ccccc}
\omega_{1,1} & \omega_{1,2} & \omega_{1,3} & 0 & \omega_{1,5} \\
* & \omega_{2,2} & 0 & 0 & 0 \\
* & * & \omega_{3,3} & \omega_{3,4} & \omega_{3,5} \\
* & * & * & \omega_{4,4} & 0 \\
* & * & * & * & \omega_{5,5}
\end{array}\right],
$$




$$
\begin{aligned}
& \Omega_{12}=\left[\begin{array}{ccccc}
\omega_{1,6} & \omega_{1,7} & \omega_{1,8} & \omega_{1,9} & \omega_{1,10} \\
0 & 0 & 0 & 0 & 0 \\
\omega_{3,6} & 0 & 0 & 0 & \omega_{3,10} \\
0 & 0 & 0 & 0 & 0 \\
\omega_{5,6} & 0 & 0 & 0 & 0
\end{array}\right], \\
& \Omega_{22}=\left[\begin{array}{ccccc}
\omega_{6,6} & 0 & 0 & 0 & 0 \\
* & \omega_{7,7} & 0 & 0 & 0 \\
* & * & \omega_{8,8} & 0 & 0 \\
* & * & * & \omega_{9,9} & 0 \\
* & * & * & * & \omega_{10,10}
\end{array}\right], \\
& \omega_{1,1}=[A+\alpha I] P+P[A+\alpha I]^{T}+(a+b+0.5 d) I-D_{1} D_{1}^{T}+d D_{1} D_{1}^{T} \\
& +0.25 D_{1} Y_{1} D_{1}^{T}+Q_{1}+Q_{2}-e^{-2 \alpha h_{1 \mathrm{~m}}} R_{1}-e^{-2 \alpha h_{1 \mathrm{M}}} R_{2}-2 e^{-4 \alpha h_{1 \mathrm{~m}}} W_{1} \\
& -2 e^{-4 \alpha h_{1 \mathrm{M}}} W_{2}-\frac{2 e^{-4 \alpha h_{1 \mathrm{M}}} h_{12}}{\left(h_{1 \mathrm{M}}+h_{1 \mathrm{~m}}\right)} W_{3}, \\
& \omega_{1,2}=P, \quad \omega_{1,3}=B P, \quad \omega_{1,5}=e^{-2 \alpha h_{1 \mathrm{~m}}} R_{1}, \quad \omega_{1,6}=e^{-2 \alpha h_{1 \mathrm{M}}} R_{2}, \\
& \omega_{1,7}=\frac{2 e^{-4 \alpha h_{1 \mathrm{~m}}}}{h_{1 \mathrm{~m}}} W_{1}, \quad \omega_{1,8}=\frac{2 e^{-4 \alpha h_{1 \mathrm{M}}}}{h_{1 \mathrm{M}}} W_{2}, \quad \omega_{1,9}=\frac{2 e^{-4 \alpha h_{1 \mathrm{M}}}}{\left(h_{1 \mathrm{M}}+h_{1 \mathrm{~m}}\right)} W_{3}, \\
& \omega_{1,10}=P A^{T}-0.5 D_{1} D_{1}^{T}, \\
& \omega_{2,2}=-\left(2 a I+Z_{1}\right)^{-1}, \quad \omega_{3,3}=-2 e^{-2 \alpha h_{1 \mathrm{M}}} R_{3}+e^{-2 \alpha h_{1 \mathrm{M}}}\left(R_{4}+R_{4}^{T}\right), \\
& \omega_{3,4}=P, \quad \omega_{3,5}=e^{-2 \alpha h_{1 \mathrm{M}}} R_{3}-e^{-2 \alpha h_{1 \mathrm{M}}} R_{4}, \\
& \omega_{3,6}=e^{-2 \alpha h_{1 \mathrm{M}}} R_{3}-e^{-2 \alpha h_{1 \mathrm{M}}} R_{4}^{T}, \quad \omega_{3,10}=P B^{T}, \\
& \omega_{4,4}=-\left(2 b I+Z_{2}\right)^{-1}, \quad \omega_{5,5}=-e^{-2 \alpha h_{1 \mathrm{~m}}} Q_{1}-e^{-2 \alpha h_{1 \mathrm{~m}}} R_{1}-e^{-2 \alpha h_{1 \mathrm{M}}} R_{3}, \\
& \omega_{5,6}=e^{-2 \alpha h_{1 \mathrm{M}}} R_{4}^{T}, \quad \omega_{6,6}=-e^{-2 \alpha h_{1 \mathrm{M}}} Q_{2}-e^{-2 \alpha h_{1 \mathrm{M}}} R_{2}-e^{-2 \alpha h_{1 \mathrm{M}}} R_{3}, \\
& \omega_{7,7}=\frac{-2 e^{-4 \alpha h_{1 \mathrm{~m}}}}{h_{1 \mathrm{~m}}^{2}} W_{1}, \quad \omega_{8,8}=\frac{-2 e^{-4 \alpha h_{1 \mathrm{M}}}}{h_{1 \mathrm{M}}^{2}} W_{2}, \quad \omega_{9,9}=\frac{-2 e^{-4 \alpha h_{1 \mathrm{M}}}}{\left(h_{1 \mathrm{M}}^{2}-h_{1 \mathrm{~m}}^{2}\right)} W_{3}, \\
& \omega_{10,10}=h_{1 \mathrm{~m}}^{2} R_{1}+h_{1 \mathrm{M}}^{2} R_{2}+h_{12}^{2} R_{3}+h_{1 \mathrm{~m}}^{2} W_{1}+h_{1 \mathrm{M}}^{2} W_{2}+h_{12} h_{1 \mathrm{M}} W_{3} \\
& +(a+b+0.5 d) I-2 P \text {. }
\end{aligned}
$$

Remark 4 From an engineering point of view, the guaranteed cost functions measured by delay state vector $x\left(t-h_{1}(t)\right)$ are more general than those where delays are not considered. For instance, congestion control on the Internet based on transmission control protocol (TCP) networks was studied in [36] and included a generally applicable nonlinear system with mixed time-varying delays. This paper is the first time when nonlinear quadratic cost functions are studied, which include both discrete and distributed delays as their performance measure for the closed-loop system. It should be noted that the proposed cost function is different from the existing literature [17, 22, 23, 29, 31]. The definition provides a more useful description, because it takes full advantage of the characteristics of distributed delay term of the nonlinear system. It is worth pointing out that the obtained exponential stability in this work is more applicable in the sense that it may still be applied to the situation when there is a distributed delay term in nonlinear quadratic cost 
functions, which can be applied in nonlinear systems with mixed time-varying delays, for example, in a population model with nonlinear death rate and distributed delays [37], and a perturbed Chua's circuit system with mixed time-varying delays [38]. Moreover, the discrete and distributed delays are not necessarily differentiable, which allows the delays to be fast time-varying functions, leading to less conservative stability criteria. In the next section, numerical examples will be given to show that our result is less conservative than some existing results in the literature [17].

\section{Numerical examples}

In this section, we present two examples to illustrate the effectiveness and the reduced conservatism of our results.

Example 4.1 Consider the nonlinear system with mixed time-varying delays via feedback control (6):

$$
\begin{aligned}
\dot{x}(t)= & A x(t)+B x\left(t-h_{1}(t)\right)+C \int_{t-d_{1}(t)}^{t} x(s) d s \\
& +f\left(t, x(t), x\left(t-h_{1}(t)\right), \int_{t-d_{1}(t)}^{t} x(s) d s, u(t)\right)+U(t), \\
U(t)= & D_{1} u(t)+D_{2} u\left(t-h_{2}(t)\right)+D_{3} \int_{t-d_{2}(t)}^{t} u(s) d s, \\
x(t)= & \phi(t), \quad t \in\left[-d_{3}, 0\right], \quad d_{3}=\max \left\{h_{1 \mathrm{M}}, h_{2}, d_{1}, d_{2}\right\},
\end{aligned}
$$

where

$$
\begin{aligned}
& A=\left[\begin{array}{ll}
0 & 0 \\
0 & 1
\end{array}\right], \quad B=\left[\begin{array}{cc}
-2 & -0.5 \\
0 & -1
\end{array}\right], \quad C=\left[\begin{array}{cc}
-0.2 & 0 \\
0 & -0.1
\end{array}\right], \\
& D_{1}=\left[\begin{array}{ll}
2 & 0 \\
0 & 3
\end{array}\right], \quad D_{2}=\left[\begin{array}{cc}
0.1 & 0 \\
0 & 0.1
\end{array}\right], \quad D_{3}=\left[\begin{array}{cc}
0.1 & 0 \\
0 & 0.1
\end{array}\right], \\
& Z_{1}=Z_{2}=Z_{3}=Y_{1}=\left[\begin{array}{cc}
0.1 & 0 \\
0 & 0.1
\end{array}\right], \quad a=b=c=d=0.001, \quad \alpha=0.2 .
\end{aligned}
$$

By using the LMI Toolbox in MATLAB, we can verify that the LMI (5) is satisfied with

$$
\begin{aligned}
& h_{1 \mathrm{~m}}=0.01, \quad h_{1 \mathrm{M}}=0.1341, \quad h_{2}=0.01, \quad d_{1}=0.05, \quad d_{2}=0.07, \\
& P=\left[\begin{array}{cc}
4.2902 & -0.7037 \\
-0.7037 & 3.9642
\end{array}\right], \quad Q_{1}=\left[\begin{array}{cc}
2.6601 & 1.1601 \\
1.1601 & 0.6259
\end{array}\right], \\
& Q_{2}=\left[\begin{array}{cc}
2.8835 & 0.3865 \\
0.3865 & 0.1653
\end{array}\right], \quad R_{1}=\left[\begin{array}{cc}
146.2015 & -353.8107 \\
-353.8107 & 919.9144
\end{array}\right], \\
& R_{2}=\left[\begin{array}{cc}
0.0340 & -0.0889 \\
-0.0889 & 0.2374
\end{array}\right], \quad R_{3}=\left[\begin{array}{cc}
23.9016 & 1.6174 \\
1.6174 & 5.6507
\end{array}\right], \\
& R_{4}=\left[\begin{array}{cc}
0.0453 & -0.1185 \\
-0.1185 & 0.3150
\end{array}\right], \quad S_{1}=\left[\begin{array}{cc}
4.6367 & -0.6837 \\
-0.6837 & 9.3435
\end{array}\right],
\end{aligned}
$$




$$
\begin{array}{ll}
T_{1}=\left[\begin{array}{cc}
0.2839 & -0.0501 \\
-0.0501 & 0.5842
\end{array}\right], & T_{2}=\left[\begin{array}{cc}
12.8421 & -2.8269 \\
-2.8269 & 4.9277
\end{array}\right], \\
W_{1}=\left[\begin{array}{cc}
8.2059 & -21.0969 \\
-21.0969 & 55.7939
\end{array}\right], & W_{2}=\left[\begin{array}{cc}
0.0456 & -0.1172 \\
-0.1172 & 0.3100
\end{array}\right], \\
W_{3}=\left[\begin{array}{cc}
0.0129 & -0.0293 \\
-0.0293 & 0.0724
\end{array}\right], & K=\left[\begin{array}{cc}
-0.2401 & -0.0426 \\
-0.0639 & -0.3897
\end{array}\right],
\end{array}
$$

and the feedback controller is

$$
u(t)=\left[\begin{array}{ll}
-0.2401 & -0.0426 \\
-0.0639 & -0.3897
\end{array}\right] x(t) .
$$

We take the initial condition $\phi(t)=\left[\begin{array}{c}0 \\ \cos t\end{array}\right],\|\phi\|=1$. Then, the upper bound on the cost function value is

$$
J^{*}=0.3254
$$

By Theorem 3.1, the system is exponential stable and solution $x(t, \phi(t))$ satisfies

$$
\| x\left(t, \phi(t) \| \leq 0.3092 e^{-0.2 t}, \quad t \geq 0 .\right.
$$

Numerical simulations are carried out using the explicit Runge-Kutta-like method (dde45), interpolation and extrapolation by splines of the third order. Figure 1 shows the trajectories $x_{1}(t)$ and $x_{2}(t)$ of nonlinear system (37) with mixed time-varying delays

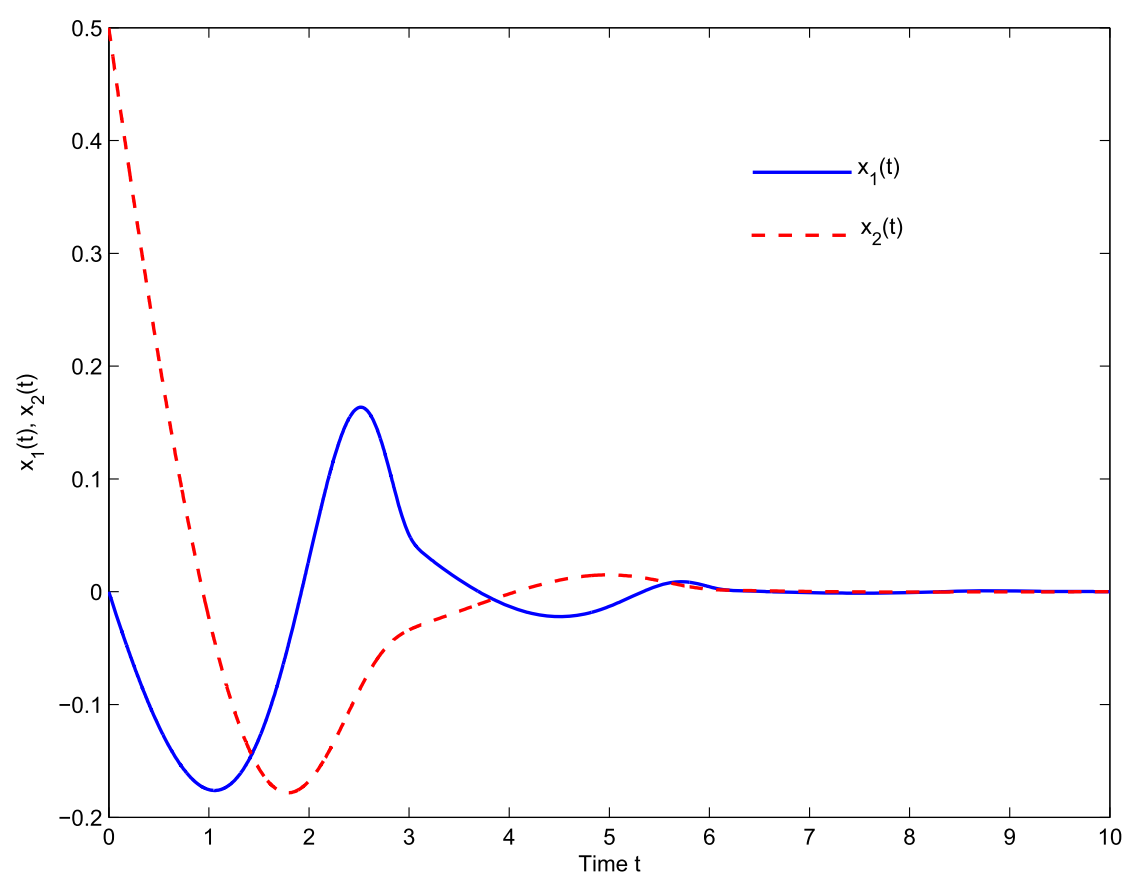

Figure 1 Trajectories $x_{1}(t)$ and $x_{2}(t)$ of nonlinear system (37) with mixed time-varying delays $h_{1}(t)=0.01+0.13|\sin t|, h_{2}(t)=0.01|\cos t|, d_{1}(t)=0.05|\cos t|$ and $d_{2}(t)=0.07|\cos t|$ and feedback control (38) 
Table 1 Maximum allowable upper bound $h_{1 \mathrm{M}}$ and an upper bound on the cost function $J^{*}$ when $\alpha=0.2$ for Example 4.1

\begin{tabular}{lll}
\hline$h_{1 \mathrm{~m}}$ & $\max h_{1 \mathrm{M}}$ & $J^{*}$ \\
\hline 0 & 0.0808 & 0.3377 \\
0.0100 & 0.1341 & 0.3254 \\
0.1300 & 0.2106 & 0.3842 \\
0.2100 & 0.2483 & 0.4221 \\
0.2400 & 0.2606 & 0.4365 \\
0.2600 & 0.2684 & 0.4461 \\
0.2700 & 0.2722 & 0.4506 \\
0.2720 & 0.2730 & 0.4516 \\
0.2730 & 0.2734 & 0.4519 \\
0.2731 & 0.2734 & 0.4523 \\
0.2732 & 0.2734 & 0.4522 \\
0.2733 & 0.2735 & 0.4520 \\
0.2734 & 0.2735 & 0.4523 \\
0.2736 & 0.2736 & 0.4522 \\
\hline
\end{tabular}

$h_{1}(t)=0.01+0.13|\sin t|, h_{2}(t)=0.01|\cos t|, d_{1}(t)=0.05|\cos t|$ and $d_{2}(t)=0.07|\cos t|$ and feedback control (38). We see that the trajectories $x_{1}(t)$ and $x_{2}(t)$ of the nonlinear system converge to zero under the above conditions. In Table 1 , we give the maximum allowable upper bounds of delay $h_{1 \mathrm{M}}$ and upper bounds on the cost function $J^{*}$ for $\alpha=0.2$ and different values of lower bounds $h_{1 \mathrm{~m}}$.

Remark 5 In Example 4.1, we show the effect of changes in the lower bound on the delay $h_{1 \mathrm{~m}}$ in computed maximum upper bounds of delay $h_{1 \mathrm{M}}$ and upper bound on the cost function. In Table 1, it can be argued that increasing the maximum upper bound on the delay leads to an increase in the upper bound on the cost function.

Example 4.2 Consider the nonlinear system with interval time-varying delay, which is described by [17]

$$
\begin{aligned}
& \dot{x}(t)=A x(t)+B x\left(t-h_{1}(t)\right)+D_{1} u(t)+f\left(t, x(t), x\left(t-h_{1}(t)\right), u(t)\right), \\
& x(t)=\phi(t), \quad t \in\left[-h_{1 \mathrm{M}}, 0\right],
\end{aligned}
$$

where

$$
\begin{aligned}
& A=\left[\begin{array}{ll}
0 & 0 \\
0 & 1
\end{array}\right], \quad B=\left[\begin{array}{cc}
-2 & -0.5 \\
0 & -1
\end{array}\right], \quad D_{1}=\left[\begin{array}{l}
0 \\
1
\end{array}\right], \\
& Z_{1}=Z_{2}=I, \quad Y_{1}=1, \quad a=b=d=0.001, \quad \alpha=0.2, \quad\|\phi\|=1 .
\end{aligned}
$$

We obtain the solution of LMI (36) for $h_{1 \mathrm{~m}}=0.01, h_{1 \mathrm{M}}=0.1155$ in Corollary 3.2 as follows:

$$
\begin{array}{ll}
P=\left[\begin{array}{cc}
0.6907 & -0.0278 \\
-0.0278 & 0.2866
\end{array}\right], & Q_{1}=\left[\begin{array}{ll}
0.0885 & 0.0686 \\
0.0686 & 0.0542
\end{array}\right], \\
Q_{2}=\left[\begin{array}{cc}
0.3128 & 0.0631 \\
0.0631 & 0.0348
\end{array}\right], & R_{1}=\left[\begin{array}{cc}
24.6092 & -7.3215 \\
-7.3215 & 3.4457
\end{array}\right], \\
R_{2}=\left[\begin{array}{cc}
0.0027 & -0.0005 \\
-0.0005 & 0.0002
\end{array}\right], & R_{3}=\left[\begin{array}{ll}
5.5719 & 0.2140 \\
0.2140 & 0.6035
\end{array}\right],
\end{array}
$$


Table 2 Maximum allowable upper bounds $h_{1 \mathrm{M}}$ and an upper bound on the cost function $J^{*}$ when $\alpha=0.2$

\begin{tabular}{|c|c|c|c|c|c|}
\hline \multicolumn{2}{|l|}{$h_{1 \mathrm{~m}}$} & \multicolumn{2}{|l|}{$h_{1 \mathrm{M}}$} & \multicolumn{2}{|l|}{$J^{*}$} \\
\hline [17] & Corollary 3.2 & {$[17]$} & Corollary 3.2 & [17] & Corollary 3.2 \\
\hline 0 & 0 & 0.0287 & 0.0657 & 289.10 & 3.6484 \\
\hline 0.0100 & 0.0100 & 0.0247 & 0.1155 & 4.0100 & 3.6542 \\
\hline 0.0200 & 0.0200 & 0.0205 & 0.1211 & 3.6900 & 3.6654 \\
\hline 0.0204 & 0.0204 & 0.0248 & 0.1213 & 3.6900 & 3.6656 \\
\hline 0.0245 & 0.0245 & 0.0248 & 0.1236 & 3.6900 & 3.6701 \\
\hline 0.0246 & 0.0246 & 0.0248 & 0.1236 & 3.6900 & 3.6703 \\
\hline 0.0247 & 0.0247 & 0.0248 & 0.1237 & 3.6900 & 3.6703 \\
\hline 0.0248 & 0.0248 & 0.0248 & 0.1237 & 3.6900 & 3.6706 \\
\hline infeasible & 0.0500 & infeasible & 0.1369 & infeasible & 3.7045 \\
\hline infeasible & 0.1000 & infeasible & 0.1595 & infeasible & 3.7792 \\
\hline infeasible & 0.1500 & infeasible & 0.1781 & infeasible & 3.8607 \\
\hline infeasible & 0.1700 & infeasible & 0.1847 & infeasible & 3.8959 \\
\hline infeasible & 0.1912 & infeasible & 0.1912 & infeasible & 3.9335 \\
\hline
\end{tabular}

$$
\begin{array}{ll}
R_{4}=\left[\begin{array}{cc}
0.0034 & -0.0007 \\
-0.0007 & 0.0002
\end{array}\right], & W_{1}=\left[\begin{array}{cc}
0.4600 & -0.0727 \\
-0.0727 & 0.0237
\end{array}\right], \\
W_{2}=\left[\begin{array}{cc}
0.0034 & -0.0005 \\
-0.0005 & 0.0002
\end{array}\right], & W_{3}=\left[\begin{array}{cc}
0.0012 & -0.0001 \\
-0.0001 & 0.0000
\end{array}\right],
\end{array}
$$

with the guaranteed cost feedback controller being $u(t)=[-0.0706-1.7517] x(t)$, and an upper bound on the cost function $J^{*}$ given by

$$
J^{*}=3.6542\|\phi\|^{2}=3.6542 \text {. }
$$

For this example, we apply Corollary 3.2 and calculate the maximum allowable upper bounds on $h_{1 \mathrm{M}}$ and minimum upper bounds on the cost function $J^{*}$ with $\alpha=0.2$ and different $h_{1 \mathrm{~m}}$. Those results are displayed in Table 2. This example shows that the proposed guaranteed cost control method gives lower guaranteed cost values than the corresponding result in [17].

\section{Conclusion and future study}

In this paper, we have investigated the problem of guaranteed cost control for exponential stability of a nonlinear system with mixed time-varying delays in state and feedback control. The mixed time-varying delays, which consisted of both interval and distributed time-varying delays, were considered without assuming the differentiability of the time-varying delays. Moreover, a novel state-feedback controller was designed which includes the state term, discrete time-varying delay term and distributed time-varying delay term for guaranteed cost control of exponential stability of the nonlinear system. Based on an improved Lyapunov-Krasovskii functional with triple integral terms, new delay-dependent sufficient conditions for the existence of guaranteed cost feedback control for the system were given in terms of linear matrix inequalities (LMIs). A performance measure for the system was considered by new nonlinear quadratic cost functions. Finally, numerical examples were given to illustrate the effectiveness and improvement over some existing results in the literature. Our goal in the future is to apply the nonlinear quadratic cost functions to other systems or networks that arise in other areas of science. 


\section{Acknowledgements}

The authors would like to express their gratitude to the editors and anonymous reviewers for their valuable suggestions, which substantially improved the standard of the paper.

\section{Funding}

The first author was financially supported by the research capability enhancement program through graduate student scholarship academic year 2016, Facutly of Science, Khon Kaen University. The second author was financially supported by the National Research Council of Thailand and Faculty of Science, Khon Kaen University 2018 and the Thailand Research Fund (TRF), the Office of the Higher Education Commission (OHEC) (grant number: MRG6080239). The fourth author was financially supported by University of Pha Yao, Pha Yao, Thailand.

\section{Competing interests}

The authors declare that they have no competing interests.

\section{Authors' contributions}

All authors contributed equally to the writing of this paper. All authors read and approved the final manuscript.

\section{Author details}

'Department of Mathematics, Khon Kaen University, Khon Kaen, Thailand. ²Department of Mathematics, Chiang Mai

University, Chiang Mai, Thailand. ${ }^{3}$ Department of Mathematics, University of Pha Yao, Pha Yao, Thailand.

\section{Publisher's Note}

Springer Nature remains neutral with regard to jurisdictional claims in published maps and institutional affiliations.

Received: 4 July 2018 Accepted: 19 November 2018 Published online: 26 November 2018

\section{References}

1. Phat, V.N.: Memoryless $H_{\infty}$ controller design for switched non-linear systems with mixed time-varying delays. Int. J. Control 82(10), 1889-1898 (2009)

2. Phat, V.N.: Switched controller design for stabilization of nonlinear hybrid systems with time-varying delays in state and control. J. Franklin Inst. 347, 195-207 (2010)

3. Phat, V.N., Botmart, T., Niamsup, P.: Switching design for exponential stability of a class of nonlinear hybrid time-delay systems. Nonlinear Anal. Hybrid Syst. 3, 1-10 (2009)

4. Zamani, I., Shafiee, M., Ibeas, A.: Exponential stability of hybrid switched nonlinear singular systems with time-varying delay. J. Franklin Inst. 350, 171-193 (2013)

5. Srinivasan, V., Sukavanam, N.: Asymptotic stability and stabilizability of nonlinear systems with delay. ISA Trans. 65, 19-26 (2016)

6. Zhang, B., Deng, F., Peng, S., Xie, S.: Stabilization and destabilization of nonlinear systems via intermittent stochastic noise with application to memristor-based system. J. Franklin Inst. 355, 3829-3852 (2018)

7. Zhao, Y., Zhang, W., Guo, W., Yu, S., Song, F.: Exponential state observers for nonlinear systems with incremental quadratic constraints and output nonlinearities. J. Control Autom. Electr. Syst. 29, 127-135 (2018)

8. Gu, K., Kharitonov, V.L., Chen, J.: Stability of Time-Delay System. Birkhäuser, Basel (2003)

9. Udwadia, F.E., Kumar, R.: Time-delayed control of classically damped structural systems. Int. J. Control 60(5), 687-713 (1994)

10. Udwadia, F.E., von Bremen, H., Kumar, R., Hosseini, M.: Time delayed control of structural systems. Earthq. Eng. Struct. Dyn. 32(2), 495-535 (2003)

11. Fridman, E., Niculescu, S.I.: On complete Lyapunov-Krasovskii functional techniques for uncertain systems with fast-varying delays. Int. J. Robust Nonlinear Control 18, 364-374 (2008)

12. Thanh, N.T., Phat, V.N.: $H_{\infty}$ control for nonlinear systems with interval non-differentiable time-varying delay. Eur. J. Control 19, 190-198 (2013)

13. Fridman, E., Shaked, U.: Delay-dependent stability and control: constant and time-varying delays. Int. J. Control 76 48-60 (2003)

14. Shao, H.: New delay-dependent stability criteria for systems with interval delay. Automatica 45, 744-749 (2009)

15. Park, J.H., Jung, H.J.: On the exponential stability of a class of nonlinear systems including delayed perturbations. J. Comput. Appl. Math. 159, 467-471 (2003)

16. Dong, Y., Liu, J.: Exponential stabilization of uncertain nonlinear time-delay systems. Adv. Differ. Equ. 2012,180 (2012)

17. Niamsup, P., Phat, V.N.: State feedback guaranteed cost controller for nonlinear time-varying delay systems. Vietnam J. Math. 43, 215-228 (2015)

18. Prasertsang, P., Botmart, T.: Novel delay-dependent exponential stabilization criteria of a nonlinear system with mixed time-varying delays via hybrid intermittent feedback control. Adv. Differ. Equ. 2017, 199 (2017)

19. Chang, S.S.L., Peng, S.S.L.: Adaptive guaranteed cost control of systems with uncertain parameters. IEEE Trans. Autom. Control 17, 474-483 (1972)

20. Moheimani, S.O., Petersen, I.R.: Optimal quadratic guaranteed cost control of a class of uncertain time-delay systems. IEE Proc., Control Theory Appl. 144, 183-188 (1997)

21. Chen, W.H., Guan, Z.H., Lu, X.: Delay-dependent output feedback guaranteed cost control for uncertain time-delay systems. Automatica 40, 1263-1268 (2004)

22. Thuan, M.V., Phat, V.N.: Optimal guaranteed cost control of linear systems with mixed interval time-varying delayed state and control. J. Optim. Theory Appl. 152, 394-412 (2012)

23. Thuan, M.V., Phat, V.N., Trinh, H.M.: Dynamic output feedback guaranteed cost control for linear systems with interval time-varying delays in states and outputs. Appl. Math. Comput. 218, 10697-10707 (2012) 
24. Yang, X., Liu, D., Wei, Q., Wang, D.: Guaranteed cost neural tracking control for a class of uncertain nonlinear systems using adaptive dynamic programming. Neurocomputing 198, 80-90 (2016)

25. Yuan, C., Dong, W., Qu, P.: Robust $H_{\infty}$ guaranteed cost control for a class of uncertain nonlinear time-delay systems. Int. J. Control Autom. 9(2), 191-198 (2016)

26. Merola, A., Cosentino, C., Colacino, D., Amato, F.: Optimal control of uncertain nonlinear quadratic systems. Automatica 83, 345-350 (2017)

27. Shen, B., Wang, Z., Tan, H.: Guaranteed cost control for uncertain nonlinear systems with mixed time-delays the discrete time case. Eur. J. Control 40, 62-67 (2018)

28. Botmart, T., Weera, W.: Guaranteed cost control for exponential synchronization of cellular neural networks with mixed time-varying delays via hybrid feedback control. Abstr. Appl. Anal. 2013, Article ID 175796 (2013)

29. Niamsup, P., Ratchagit, K., Phat, V.N.: Novel criteria for finite-time stabilization and guaranteed cost control of delayed neural networks. Neurocomputing 160, 281-286 (2015)

30. He, H., Yan, L., Tu, J.: Guaranteed cost stabilization of time-varying delay cellular neural networks via Riccati inequality approach. Neural Process. Lett. 35, 151-158 (2012)

31. Rajchakit, G.: Delay-dependent optimal guaranteed cost control of stochastic neural networks with interval nondifferentiable time-varying delays. Adv. Differ. Equ. 2013, 241 (2013)

32. Lee, T.H., Park, J.H., Ji, D.H., Kwon, O.M., Lee, S.M.: Guaranteed cost synchronization of a complex dynamical network via dynamic feedback control. Appl. Math. Comput. 218, 6469-6481 (2012)

33. Lee, T.H., Ji, D.H., Park, J.H., Jung, H.Y.: Decentralized guaranteed cost dynamic control for synchronization of a complex dynamical network with randomly switching topology. Appl. Math. Comput. 219, 996-1010 (2012)

34. Li, S., Tang, W., Zhang, J.: Guaranteed cost control of synchronisation for uncertain complex delayed networks. Int. J. Syst. Sci. 43, 566-575 (2004)

35. He, P., Wang, X.-L., Li, Y.: Guaranteed cost synchronization of complex networks with uncertainties and time-varying delays. Complexity 21, 381-395 (2015)

36. Wang, X.F., Chen, G.R., Ko, K.T.: A stability theorem for Internet congestion control. Syst. Control Lett. 43, 81-85 (2002)

37. El-Morshedy, H.A.: Global attractivity in a population model with nonlinear death rate and distributed delays. J. Math. Anal. Appl. 410, 642-658 (2014)

38. Niamsup, P., Botmart, T., Weera, W.: Modified function projective synchronization of complex dynamical networks with mixed time-varying and asymmetric coupling delays via new hybrid pinning adaptive control. Adv. Differ. Equ. $2017,124(2017)$

\section{Submit your manuscript to a SpringerOpen ${ }^{\circ}$ journal and benefit from:}

- Convenient online submission

- Rigorous peer review

- Open access: articles freely available online

- High visibility within the field

- Retaining the copyright to your article

Submit your next manuscript at $\gg$ springeropen.com 